\title{
Spectroscopic Investigations for Photo Stability of Diclofenac Sodium Complexed with Hydroxypropyl- $\beta$-Cyclodextrin
}

\author{
Alagumuthu Manikandan, Shubhada Chandrasekhar Nemani, V. Sadheeshkumar, Sivakumar Arumugam* \\ School of Bio-Sciences and Technology, VIT University, Vellore, Tamil Nadu, India-632014., Center of Advanced Study in Marine Biology, Annamalai \\ University, India.
}

\begin{tabular}{|c|c|}
\hline ARTICLE INFO & ABSTRACT \\
\hline Article history: & \multirow{10}{*}{$\begin{array}{l}\text { Diclofenac sodium was complexed with hydroxypropyl- } \beta \text {-cyclodextrin and physicochemical characterization } \\
\text { was performed to evaluate the photo stability of diclofenac sodium. X-ray powder diffraction, UV-Vis analysis } \\
\text { and Fourier Transform Infrared Spectroscopy were performed to examine the changes if any in the morphology } \\
\text { and the configuration of the complex. The photo stability test followed by High Performance Liquid } \\
\text { Chromatography analysis of the complex was determined. In vitro anti-inflammatory test using Human Red } \\
\text { Blood Cell membrane stabilization method and in vivo analgesic activity studies in rat models to evaluate the } \\
\text { therapeutic potential assessment of the complexed and norml diclofenac sodium. The test results have proved } \\
\text { that the complexed diclofenac with hydroxypropyl- } \beta \text {-cyclodextrin enhance the photodegradation rate and } \\
\text { resolute the optimal molar ratio of diclofenac to hydroxypropyl- } \beta \text {-cyclodextrin as } 1: 4 \text {. In vitro anti-inflammatory } \\
\text { activity and in vivo analgesic activity results are indicates that the complexed form of diclofenac sodium with } \\
\text { hydroxypropyl- } \beta \text {-cyclodextrin does not affects the therapeutic potentials of the drug. }\end{array}$} \\
\hline Received on: 30/01/2016 & \\
\hline Revised on: $16 / 02 / 2016$ & \\
\hline Accepted on: 07/03/2016 & \\
\hline Available online: $30 / 04 / 2016$ & \\
\hline Key words: & \\
\hline Photo stability, Diclofenac & \\
\hline Sodium, hydroxypropyl- $\beta$ - & \\
\hline cyclodextrin, Anti- & \\
\hline inflammation, analgesic & \\
\hline
\end{tabular}

\section{INTRODUCTION}

Diclofenac sodium [Benzeneacetic acid,2- $[(2,6-$ dichlorophenyl)amino]-,monosodium salt] is a synthetic nonsteroidal anti-inflammatory drug (NSAID) with analgesic, antiinflammatory and antipyretic activity. Its mechanism of action is associated mainly with the inhibition of prostaglandin synthesis (specifically, inhibition of cyclooxygenase) available in oral, topical and parenteral preparations (Dastidar et al., 2000). Diclofenac is often used in the treatment of chronic pain linked with cancer, particularly in if inflammation involved diseases. Diclofenac is similar in COX-2 selectivity to celecoxib (FitzGerald and Patrono, 2001). Diclofenac generate unfavourable effects in about $20 \%$ of patients, and about $2 \%$ patients withdrawing treatment as a result (Burk and Smyth,

* Corresponding Author

Sivakumar Arumugam, School of Bio-Sciences and Technology, VIT

University, Vellore, Tamil Nadu, India. Email: siva_kumar.a@vit.ac.in
2006). On the whole of the unfavourable drug responses are accounted with the oral or parenteral use of diclofenac like nausea, epigastric pain, hypersensitivity reactions like urticaria, peptic ulcer, erythema multiforme, impaired renal function, dizziness, elevation of serum transaminase, angioedema, bullous eruption, allergic purpura, toxic epidermal necrolysis, and Steven-Johnson syndrome (Taylor et al., 2011). A rigorous photosensitivity reaction caused by topical preparation of diclofenac sodium was already reported (Akath, 2013). Cyclodextrins (CDs) are widely used in chemical industries food, pharmaceutical (Stéphane et al., 2007), and drug delivery (Thimma et al., 2010). They are classified as cyclic oligoglycosides of 6-( $\alpha-C D), 7-(\beta-C D)$, or 8-( $\gamma-C D)$. $\alpha$ D-glucopyranose units have circular configuration which results in a donut-shaped molecule in solution that has a hydrophilic exterior and a hydrophobic interior. This cavity is capable of binding guest molecules of the appropriate size, shape, and polarity, such as surfactant tails, dyes, drugs, etc., forming non-covalently bonded inclusion complexes (Junquera et al., 1995). 
The tendency of CDs to house guest molecules results in two very important properties. One is their capability to protect, stabilize or solubilise guest molecules; the other is their ability to selectively orient them. As CDs have no toxic effects, the first capability is of great value to the food, drug and agricultural industries (Volobuef et al., 2012). The complexation of DS with HP $\beta C D$ (hydroxypropyl- $\beta$-cyclodextrin) would be interesting because of its improved complexing ability, water solubility and lower toxicity than that of $\beta-C D$, changing the pharmacological properties of this analgesic and thus providing it the photo stability. Hisham et al. 2015 used chromatographic (HPLC) investigations for simultaneous determination of atenolol and nifedipine in presence of atenolol pharmacopeoial impurities.

In this study, the development of an insertion complex linking Diclofenac and HP $\beta C D$ was studied and improved photo stability activities of diclofenac in the existence of HP $\beta C D$ was examined using XRD, UV-Vis, FTIR and HPLC. The antiinflammatory and analgesic evaluation results for the postactivities of diclofenac sodium and the complexed DS+HP $\beta C D$ prepared in various ratios were also discussed.

\section{MATERIALS AND METHODS}

\section{Reagents}

Diclofenac Sodium was a legacy sample from Blessing Pharmaceuticals India (Maharashtra, India); HP $\beta C D$ was a legacy sample from Gangwal Chemicals (Maharashtra, India). Sodium Lauryl Sulphate and de-ionized water were from VIT University, Vellore India.

\section{Preparation of physical mixtures}

Uncomplexed diclofenac sodium and HP $\beta C D$ were weighed to make the mixtures in four different ratio of $2: 1,1: 1$, $1: 2$ and 1:4. The sample powders were mixed vigorously in a beaker with a glass rod. After thorough mixing, the mixtures of all the four ratios were gathered and labelled accordingly. Inclusion complex of diclofenac sodium (DS) and HP $\beta C D$ was weighed to make the mixtures in four different ratios of 2:1, 1:1, 1:2 and 1:4. Demineralized water was added to DS samples, which were followed by sodium lauryl sulphate, a solubilising agent. Finally, HP $\beta C D$ was added to this mixture. The mixture was shaken vigorously and kept overnight. Some amount of the inclusion complexes was freeze dried, while the rest was filtered using a Whatman filter paper to obtain clear solutions.

\section{Physiochemical characterization studies}

Physiochemical characterizations like XRD, FTIR and HPLC were performed in VIT University, India. For powder XRD analysis, powder diffractograms of DS, HP $\beta C D$, physical mixtures and the inclusion complexes were collected. The particulars for DS were $2 \theta=5^{\circ}-60^{\circ}$, the tube voltage of $40 \mathrm{~kW}$ and the tube current of $30 \mathrm{~mA}$, the step size was $0.02^{\circ}$ and the time frame was $0.02^{\circ}$ per second. For $\mathrm{HP} \beta C D$ it was $2 \theta=5^{\circ}-60^{\circ}$ and the angle range was $2^{\circ}-70^{\circ}$ (Bing-Xin et al., 2012, Klaus-Dieter et al., 2003). Fourier Transform Infrared Spectroscopy (FTIR) experiments were carried out in Shimadzu, IR Affinity-1 spectrometer in a range from 400 to $4000 \mathrm{~cm}^{-1}$ with $2 \mathrm{~cm}^{-1}$ resolution. DS, HPßCD, physical mixtures and the inclusion complexes were analyzed using $\mathrm{KBr}$ pellets (Ghodke et al., 2010). UV-Visible Analysis were carried out in a Jasco, V-670 spectrometer at $276 \mathrm{~nm}$ in a range of $200-400 \mathrm{~nm}$ for DS and at 282nm in a range of $250-350 \mathrm{~nm}$ for HP $\beta C D$.

The photo stability of DS was assessed in the presence and absence of HP $\beta C D$. The clear solutions of different ratios, prepared from the inclusion complexes, were positioned $50 \mathrm{~cm}$ away from a UV light source. They were exposed to UV light for four different time intervals- $0,2,4$ and 6 hours. DS concentrations were determined by HPLC.

\section{In-Vitro anti-inflammatory activity}

In-vitro Anti-Inflammatory activity was performed by Human Red Blood Cell (HRBC) membrane stabilization method using diclofenac sodium as standard (Ejebe et al., 2010, Manikandan et al., 2015). The percentage haemolysis was calculated by assuming the haemolysis produced in the presence of distilled water at $100 \%$. The percentage of HRBC membrane stabilization was calculated using the following formula,

$\%$ Inhibition of haemolysis $=100 \mathrm{x}$ [(OD1-OD2)/OD1], Where OD2 = optical density of sample OD1 = optical density of control.

\section{In vivo analgesic activity evaluations}

Wistar albino rats of either sex, $150 \pm 10 \mathrm{~g}$, were procured from the animal house, Annamalai University, India. They were in a controlled room with a $12 \mathrm{~h}$ dark-light cycle and fed with commercial pellet feed from Hindustan Lever Ltd. (Mumbai, India); water was freely available. Animal model study was approved (Vide No.1038, 2013) by the Institutional Animal ethics committee of Annamalai University, India and was conducted in accordance with the "Guidelines for care and Use of Laboratory Animals", Government of India.

\section{Test of peripheral analgesic activity by aceticacid-induced writhing response}

The acetic acid-induced writhing test was carried out in accordance with (Collier et al. 1968). The rats were intraperitoneally injected with $0.6 \%$ acetic acid $(10 \mathrm{~mL} / \mathrm{kg}$ b.wt) to elicit writhing response. Immediately after the administration of acetic acid, the animals were placed inglass cages and the number of writhes was recorded for the following $30 \mathrm{~min}$.

A significant reduction in the number of writhes by drug treatment as compared to control animals was considered as a positive analgesic response. Diclofenac sodium and complexed form of diclofenac sodium with HP $\beta C D$ (50 mg/kg b.wt) and indomethacin $(10 \mathrm{mg} / \mathrm{kg} \quad$ b.wt $)$ were administered intraperitoneally $30 \mathrm{~min}$ before the acetic acid injection. 


\section{Test of central analgesic activity of $p$-CA ( $p$-Coumaric acid) by tail-immersiontest}

The tail-immersion test was carried out as described by (Janssen et al. 1963), was immersed in a water bath thermostatically maintained at $55 \pm 1^{\circ} \mathrm{C}$. The time in seconds for the tail withdrawal from the water was taken as the tail withdrawal latency orreaction time, with a cut-off time of immersion set at 10 seconds. The reaction time was measured before drug treatment at $15,30,45$ and 60 minutes after the drugs were administered. Diclofenac sodium and complexed form of diclofenac sodium with $\mathrm{HP} \beta C D(50 \mathrm{mg} / \mathrm{kg}$ b.wt) and indomethacin $(10 \mathrm{mg} / \mathrm{kg}$ b.wt $)$ were administered at lower $5-\mathrm{cm}$ portion of the tail.

\section{Statistical Analysis}

All in vitro and in vivo experiment results were expressed as percentage decrease with respect to control values and compared by one-way ANOVA with Dunnett's post test was performed. GraphPad Prism version 6.07 for Windows, GraphPad Software, San Diego California USA, www.graphpad.com was used for statistical analysis. A difference was considered statistically significant if $p \leq 0.05$.

\section{RESULTS AND DISCUSSIONS}

\section{Physicochemical characterization}

For inclusion complex and physical mixture the following codes were given as respectively ratio $2: 1-1 \&$ A, ratio 1:1 - 2 \& B, ratio 1:2 - $3 \&$ C, ratio 1:4 - 4 \& D, Diclofenac sodium as DS and Hydroxypropyl- $\beta$-cyclodextrin as H (Refer supplementary file for all spectrum figures). Physicochemical characterization was performed by executing $\mathrm{X}$-ray powder diffraction (XRD), UV-Vis analysis, Fourier Transform Infrared Spectroscopy (FTIR) and the photo stability test followed by High Performance Liquid Chromatography (HPLC) analysis of the complex was carried out (Refer supplementary file for all result spectrum figures).

\section{XRD analysis}

The powder diffractograms from HP $\beta C D$, DS, physical mixture, and the inclusion complex were performed on a $\mathrm{X}$-ray diffractometer with a $\mathrm{Cu}-\mathrm{K} \alpha$ radiation source and the tube voltage and current set to $40 \mathrm{~kW}$ and $30 \mathrm{~mA}$, respectively, operated at a scan rate of $0.02^{\circ} /$ second, between $2 \theta=5^{\circ}$ and $60^{\circ}$ in a $\theta-2 \theta$ configuration. The DS diffractogram reveals a crystalline nature, whereas $\mathrm{HP} \beta C D$ is amorphous. For the ratio 2:1, the amount of DS is twice as that of $\mathrm{HP} \beta C D$. The pattern of the physical mixture was almost similar as DS with some similarities with HP $\beta C D$ pattern. But, the pattern was more inclined towards DS than HP $\beta C D$. The inclusion complex gives a pattern which is somewhat similar to the DS pattern. But the pattern also shows some amorphous nature, which indicates that the complexation was complete. For the ratio $1: 1$, the amount of DS and $\mathrm{HP} \beta C D$ was equal. The physical mixture of DS and HP $\beta C D$ gives a superposition of crystalline DS and the amorphous HP $\beta C D$. On the contrary, the inclusion complex gives a slightly different pattern from the crystalline DS. In addition, a similar pattern as observed in the diffractogram related to $\mathrm{HP} \beta \mathrm{CD}$, which suggests conformation changes for DS in the inclusion complex.

The partial loss of crystallinity was observed. So, the inclusion complex was partially amorphous. But since the pattern was partial both sides, it can be said that the reduction in the intensity was $50 \%$ than pure $\mathrm{HP} \beta C \mathrm{CD}$, showing a complete complexation of the DS. For ratio 1:2, the amount of HP $\beta C D$ was twice as DS. The pattern of the physical mixture was similar as $\mathrm{HP} \beta C D$, which shows that the complexation was complete and DS have combined with HP $\beta C D$. The inclusion complex pattern, also, was similar to HP $\beta C D$ pattern. This may be because the amount of $\mathrm{HP} \beta C D$ was higher than DS. But, from the patterns it is obvious that both the physical mixture and the inclusion complex were amorphous in nature. For the ratio $1: 4$, the amount of $\mathrm{HP} \beta C D$ was 4 times the amount of DS. The pattern of the physical mixture and that of the inclusion complex were same as that of HP $\beta C D$. This clearly indicates that both of them were of amorphous nature. $\mathrm{HP} \beta C \mathrm{CD}$ was attached to DS, thus, creating steric hinderance. So, due to the steric hinderance the crystallinity properties of DS were masked and they appeared to be amorphous.

\section{FTIR analysis}

DS showed peaks at $2970(\mathrm{~N}-\mathrm{H}), 1575(\mathrm{~N}-\mathrm{H}), 1556$ $(\mathrm{C}=\mathrm{O})$ and $715(\mathrm{C}-\mathrm{H}) \mathrm{cm}^{-1}$. HP- $\beta-\mathrm{CD}$ infrared spectrum presented at 2929 and $1157 \mathrm{~cm}^{-1}$ corresponding to the $\mathrm{NH}$ and $\mathrm{C}-\mathrm{O}$ groups, respectively. Other significant peaks for DS were 3385, 3035, $1498,1282,844,765$ and $746 \mathrm{~cm}^{-1}$ whereas for HP- $\beta-C D$ were $1332,1082,707$ and $582 \mathrm{~cm}^{-1}$. For the ratio $2: 1$, inclusion complex peaks for DS were 3385, 1575, 1556 and $715 \mathrm{~cm}^{-1}$ and from HP- $\beta$ CD was $2965 \mathrm{~cm}^{-1}$. For physical mixture, the peaks for DS were $3035,2970,1575,1556,1498,1282,844,765,746$ and $715 \mathrm{~cm}^{-1}$ and from HP- $\beta$-CD were 2929 and $1157 \mathrm{~cm}^{-1}$. In the ratio 1:1, for the inclusion complex, the peaks from DS were $3385,3035,2970$, $1575,1556,1498,1467,1282,844,765,746,715$, and $559 \mathrm{~cm}^{-1}$ and from HP- $\beta-C D$ were 2929,1157 and $582 \mathrm{~cm}^{-1}$. For physical mixture, the peaks from DS were $3035,2970,1575,1556,1498$, $1467,1282,1192,1166,844,765,746$ and $715 \mathrm{~cm}^{-1}$ and from HP$\beta$-CD were 2929 and $1041 \mathrm{~cm}^{-1}$. For the ratio $1: 2$, the inclusion complex peaks from DS were 3385 and $2970 \mathrm{~cm}^{-1}$ and from HP- $\beta$ CD were 2929, 1641, 1332, 1157, 1082, 854, 707, 582 and 547 $\mathrm{cm}^{-1}$. For physical mixture, the peaks from DS were 2970, 1556, $1452,1282,844,765,715$ and $559 \mathrm{~cm}^{-1}$ and from HP- $\beta-C D$ were 2065 and $1157 \mathrm{~cm}^{-1}$.

In the ratio 1:4, the inclusion complex peaks from DS were 2970 and 1303 and from HP $\beta C D$ were 2929, 1641, 1332, $1157,1082,947,854$ and $707 \mathrm{~cm}^{-1}$ and for physical mixture the peaks from DS were $3385,2970,1575,1556,1498,1467,1303$, $1282,844,765,746$ and $715 \mathrm{~cm}^{-1}$ and from HPßCD was 1157 $\mathrm{cm}^{-1}$. The analysis of DS- HP- $\beta-C D$ for ratio 1:1 showed that all the significant peaks from both DS and $\mathrm{HP} \beta C D$ were present which clearly indicates the association of DS with HP $\beta C D$. The sensitivity of this technique is to detect the drug in the 
concentration that they were used in the formulations, the infrared spectra for DS was recorded using the mixtures of drug and HP $\beta C D$ at the proportions of $2: 1,1: 1,1: 2$ and 1:4 (w/w), showing now for Fourier Transform Infrared Spectroscopy (FTIR) analysis that the characteristics peaks of DS and HP $\beta C D$ could be detected in the curves at the concentration used in the complex preparations. Some peaks were observed near the peak values of DS and HP $\beta C D$ with a difference of $(+)$ or $(-) 2$.

\section{UV-Vis analysis}

For confirmatory studies, samples were exposed to light providing an overall illumination of not less than 1.2 million lux hours and an integrated near ultraviolet energy of not less than 200 watt hours/square meter to allow direct comparisons to be made between diclofenac and the complexes in various ratio. The analysis of UV was performed with the help of XRD data. From the XRD patterns of DS and the complexes, it was observed that DS was retained in the complexes after complexation was completed. This resulted in a strong peak at 6.639 in the XRD pattern of DS. A peak at 21.930 was observed in the complex patterns. It was the first strongest peak of the complex. This was because there was no peak in the DS XRD pattern at 21.930. This indicates that this strong peak in the complex patterns can only come from a complex. These observations showed that the complete amount of DS was never utilized even after complexation, irrespective of the HP $\beta C D$ amounts in the ratio. So, the peaks observed in the UV graph is because of the retained DS in the complexes. Here, a possibility arises that the peak of the complex lies outside the decided range for UV. And since DS is retained in the complexes, both inclusion complex and the physical mixture, a strong peak is observed in the XRD pattern for DS and not for the complexes.

Since, the XRD peak height is proportional to the weight percentage of the sample, a strong peak of DS was observed in the pattern of the complexes. The conclusion drawn from this analysis is that the ratios were complexed and efficiently completed. The peaks for DS as per the strength of the peak are at 6.639, 8.514, 15.108 and 23.409 whereas the strongest peak of the complex was observed at 21.930. A clear conclusion from UV analysis can be drawn which is that there is some DS always retained in the complexes after complexation. This is observed from a constant peak value in UV results. This is probably because the molecule size of DS is more than that of HP $\beta C D$. So, there is steric hinderance to the active sites of the molecule which is why more complexation is not attained. The future perspective for this would be to reduce the concentration of DS or to change the complexing agent or to change the method of synthesis.

\section{HPLC analysis}

Complexed diclofenac in aqueous solution was measured by HPLC (Shimadzu LC-20A pump, Shim-pack VP-DDS-C18 column $(4.6 \times 150 \mathrm{~mm}, 3 \mu \mathrm{m}))$ at a flow rate of $0.8 \mathrm{~mL}$ min-1 and using a UV detector (SPD-20A detector) at $245 \mathrm{~nm}$. The injection volume was $10 \mu \mathrm{L}$. The eluent comprised $60 \%$ methanol and $40 \%$ deionized water $(\mathrm{pH}=2.5)$. Samples of all the ratios were exposed to UV light for 4 different time intervals- 0, 2, 4 and 6 hrs. Each sample responded differently to these conditions. The retention time for each sample was different. It decreased with the increasing time interval and UV exposure. It was also observed that the area of the peak was reduced with increasing time interval and UV exposure. The values for the ratios 2:1, 1:2 and 1:4 are decreasing with increasing time interval and UV exposure. This means that the amount of DS decreased in each of the samples after exposure to light. The values for DS are also decreasing with increasing time interval and UV exposure which indicates that DS degraded when exposed to light. But, in case of ratio $1: 1$, the values decreased at first but then increased at the end. This was because DS was photo-stabilized due to HP $\beta C D$ complexation. The amount released after 6 hrs was more than that after 4 hrs (Table-1) which is clearly indicated the photo-stabilization. Figure- 1 clearly illustrating photostabilization of complexes by showing the complex ratio values decreasing with increasing time interval and UV exposure. The ratio 1:4 is showing remarkable photostabilization among all the complexes.

Table 1: HPLC results for photo stability.

\begin{tabular}{ccccc}
\hline Ratio / Time & $\mathbf{0 ~ h r s}$ & $\mathbf{2}$ hrs & $\mathbf{4}$ hrs & $\mathbf{6}$ hrs \\
\hline $2: 1$ & $\mathbf{1}$ & $\mathbf{6}$ & $\mathbf{1 1}$ & $\mathbf{1 6}$ \\
\% Release & 117.24 & 60.02 & 54.41 & 48.24 \\
$1: 1$ & $\mathbf{2}$ & $\mathbf{7}$ & $\mathbf{1 2}$ & $\mathbf{1 7}$ \\
\% Release & 90.95 & 48.87 & 42.92 & 53.55 \\
$1: 2$ & $\mathbf{3}$ & $\mathbf{8}$ & $\mathbf{1 3}$ & $\mathbf{1 8}$ \\
\% Release & 52.45 & 34.50 & 30.29 & 27.20 \\
$1: 4$ & $\mathbf{4}$ & $\mathbf{9}$ & $\mathbf{1 4}$ & $\mathbf{1 9}$ \\
\% Release & 32.47 & 29.92 & 20.23 & 18.02 \\
DS* & $\mathbf{5}$ & $\mathbf{1 0}$ & $\mathbf{1 5}$ & $\mathbf{2 0}$ \\
\% Release & 85 & 81.8 & 71.4 & 56.1
\end{tabular}

*DS-Diclofenac sodium, Numbers in bold indicating the reading in time interval

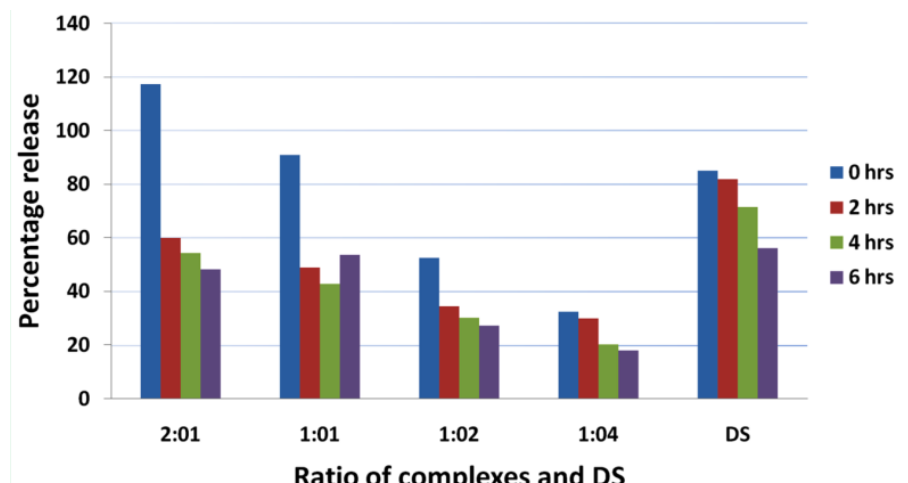

Fig. 1: Complex ratio values decreasing with increasing time interval and UV exposure.

\section{In-Vitro anti-inflammatory activity}

As per the results set out in Table 4, the maximum values were exposed only at the higher concentration. From this, it was understood that the anti-inflammatory activity of complexed samples is dose depended. The calculated \% inhibition indicates that the samples in the ratio 1:4 and 1:2 showing best activity when compared to rest complexed samples while the ratio 1:1 showed a moderate activity. Also the $\mathrm{IC}_{50}$ values are almost equal to the ratio of 1:4 with standard diclofenac. The $\mathrm{IC}_{50}$ value was a 
little bit less than standard for the ratio $1: 2$ indicate its considerable efficiency in anti-inflammatory activity.

Table 2: In-vitro anti-inflammatory activity of complexes diclofenac as standard.

\begin{tabular}{ccccc}
\hline Test samples & Mean \pm SEM & R square & $\boldsymbol{p}$-value & $\mathbf{I C}_{\mathbf{5 0}}$ \\
\hline Diclofenac sodium & $90.35 \pm 9.23^{* *}$ & 0.986 & 0.0103 & 24.983 \\
$2: 1$ & $72.03 \pm 6.46^{* *}$ & 0.919 & 0.0268 & 42.066 \\
$1: 1$ & $78.98 \pm 7.15^{* *}$ & 0.912 & 0.0197 & 43.579 \\
$1: 2$ & $87.72 \pm 7.84^{* *}$ & 0.923 & 0.0166 & 25.897 \\
$1: 4$ & $88.73 \pm 3.59^{* *}$ & 0.975 & 0.0125 & 25.023 \\
\hline
\end{tabular}

*Data analyzed by one way ANOVA followed by Dunnett's't' test, $(\mathrm{n}=3),{ }^{* *}$ $p<0.05$ significant from control.

\section{In vivo analgesic activity evaluations \\ Result of peripheral analgesic activity by aceticacid-induced writhing response}

In the acetic acid-induced writhing test, diclofenac sodium complexes showed significant peripheral analgesic activity in a dose-dependent manner, as depicted in Figure-2. Intraperitoneal injection of acetic acid in rats significantly increased the writhing. Among all the complexes, 1:4 ratio complex showing almost equal activity when compare with the diclofenac sodium while 1:2 showing a moderate.

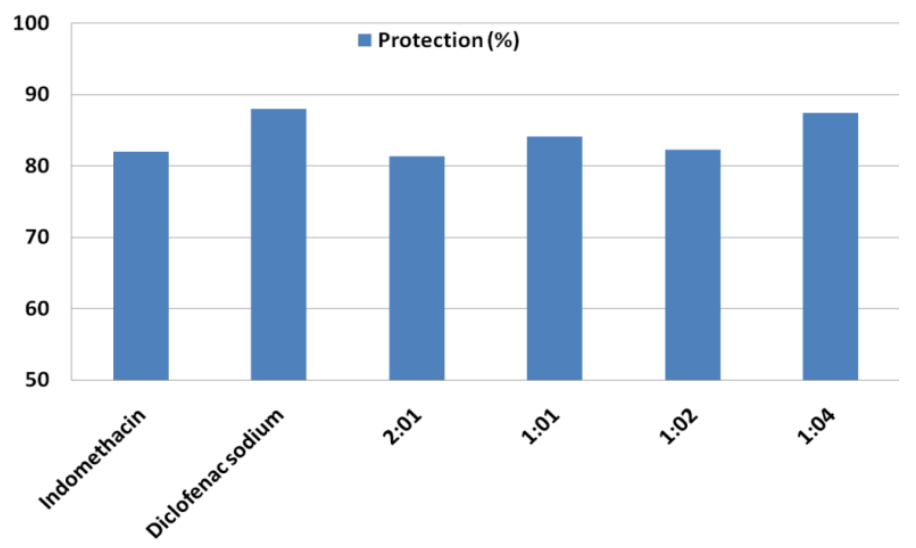

Fig. 2: Effect of samples on acetic acid-induced writhing response in rat.

Table 3: Effect of diclofenac and its complex at various ratio and indomethacin on tail-immersion test in rats.

\begin{tabular}{lll}
\hline \multicolumn{1}{c}{ Test samples } & \multicolumn{1}{c}{ Mean writhing $(\mathbf{X} \pm \mathbf{S E})$} & Protection (\%) \\
\hline Control & $28.0 \pm 1.55^{* *}$ & - \\
Indomethacin & $4.4 \pm 1.46^{* *}$ & 82.00 \\
Diclofenac sodium & $3.4 \pm 2.44^{* *}$ & 88.00 \\
$2: 1$ & $5.8 \pm 1.55^{* *}$ & 81.33 \\
$1: 1$ & $4.0 \pm 1.22^{* *}$ & 84.12 \\
$1: 2$ & $5.4 \pm 2.41^{* *}$ & 82.33 \\
$1: 4$ & $3.7 \pm 1.12^{* *}$ & 87.48 \\
\hline
\end{tabular}

Data represent mean values \pm SE of six mice per group, shown at the final value for each group. Comparisons are made with the control group. **Symbols represent statistical significance when $p<0.05$

\section{Result of central analgesic activity of $p$-CA ( $p$-Coumaric acid) by tail-immersiontest}

Table-2 below depicts the central analgesic activity of diclofenac and its complex as measured by the tail-immersion test. Diclofenac (50/100 mg/kg b.wt) exhibited significant analgesic activity in a dose-dependent manner by delaying the tail withdrawal latency or reaction time, compared to the control group. Indo-methacin treatment was found to have a better effect compared to diclofenac treatment. Among all the complexes, 1:4 showed a maximum activity, 1:1 and 1:2 ratio complexes showed almost equal activity while 1:2 showing a moderate when compare with the diclofenac sodium.

\section{CONCLUSION}

In this study, photo stability of diclofenac sodium, and its liquid inclusion complexes with HP $\beta C D$, was studied in aqueous solution. XRD pattern, FTIR spectra, UV-absorption spectra and HPLC of diclofenac sodium and inclusion complexes, physical mixtures in various concentrations were determined. One of the best concentrations of the products was identified as 1:4. Complexation of diclofenac sodium in the presence of $\mathrm{HP} \beta C D$ leads to a more enhanced photo stability, an effect which could be due to the activities of complexed radical intermediates. In vitro anti-inflammatory and in vitro analgesic activity results confirmed that there is no convertion in the normal activity of diclofenac sodium when it was complexed with HP $\beta C D$. It was understood from all investigations that diclofenac sodium complexed with hydroxypropyl- $\beta$-cyclodextrin enhances the photo stability of diclofenac sodium by not affecting the normal therapeutic potentials.

\section{ACKNOWLEDGEMENTS}

The authors are grateful for the Sophisticated Instrument Facility (SIF), School of Bio-Science and Technology, VIT University, India for providing necessary laboratory facilities and financial support.

\section{REFERENCES}

Akat PB. Severe photosensitivity reaction induced by topical diclofenac. Indian Journal of Pharmacol, 2013; 45: 408-409.

Bing-Xin Wei, Lin Zhao, Ting-Jie Wang, Han Gao, Hai-Xia $\mathrm{Wu}$, Yong Jin. Photo-stability of $\mathrm{TiO} 2$ particles coated with several transition metal oxides and its measurement by rhodamine-B degradation. Advanced Powder Technology, 2013; 24: 708-713.

Burke A, Smyth E, FitzGerald GA, 2006. Analgesic-antipyretic agents; pharmacotherapy of gout. The Pharmacological Basis of Therapeutics. $11^{\text {th }}$ ed. New York: McGraw Hill Medical Publishing Division. 671-715.

Collier HO, Dinneen LC, Johnson CA, Schneider C. The abdominal constriction response and its suppression by analgesic drugs in the mouse. Br J Pharmacol Chemother, 1968; 32: 295-310.

Dastidar SG, Ganguly K, Chaudhuri K, Chakrabarty AN. The anti-bacterial action of diclofenac shown by inhibition of DNA synthesis. Int. J. Antimicrob. Agents, 2000; 14: 249-251.

Ejebe DE, Siminialayi IM, Emudainowho JOT, Ofesi U, Morka L. Analgesic and anti inflammatory activities of the ethanol extract of the leaves of Helianthus annus in wistar rats. Asian Pac J Trop Med, 2010; 3 : 341-347.

FitzGerald GA, Patrono C. The coxibs, selective inhibitors of cyclooxygenase-2. N Engl J Med, 2001; 345: 433-442.

Ghodke DS, Chaulang GM, Patil KS, Nakhat PD, Yeole PG, Naikwade NS, and Magdum CS. Solid State Characterization of 
Domperidone: Hydroxypropyl- $\beta$-Cyclodextrin Inclusion Complex, Indian J Pharm Sci. 2010; 72: 245-249.

Hisham Hashem, Ibrahim Adel Ehab, Elhenawee Magda. A novel stability indicating HPLC-method for simultaneous determination of atenolol and nifedipine in presence of atenolol pharmacopeoial impurities. Journal of Applied Pharmaceutical Science, 2015; 5:17-25.

Janssen PA, Niemegeers CJ, Dony JG. The inhibitory effect of fentanyl and other morphine-like analgesics on the warm waterinduced tail withdrawal reflex in rats. Arzneimittelforschung, 1963; 13: 502-07.

Junquera E, Pena L, Aicart E. A Conductimetric Study of the interaction of Hydroxypropyl- $\beta$-cyclodextrin with Dodecyltrimethylammonium Bromide in water solution. Langmuir, 1995; 11: 4685-90.

Liss Klaus-Dieter, Bartels Arno, Schreyer Andreas, Clemens Helmut. High-Energy X-Rays: A tool for Advanced Bulk Investigations in Materials Science and Physics. Textures and Microstructures, 2003; 35: 219.

Manikandan A, Sathiyanarayanan KI and Sivakumar A. Molecular docking, design, synthesis, in vitro antioxidant and antiinflammatory evaluations of new isoquinoline derivatives, Int J Pharm Pharm Sci, 2015; 7: 200-208.

Menuel Stéphane, Joly Jean-Pierre, Courcot Blandine, Elysée Josias, Ghermani Nour-Eddine, Marsura Alain. Synthesis and inclusion ability of a bis- $\beta$-cyclodextrin pseudo-cryptand towards Busulfan anticancer agent. Tetrahedron, 2007; 63: 1706-14.
Taylor RS, Fotopoulos G, Maibach H. Safety profile of topical diclofenac: A meta-analysis of blinded, randomized, controlled trials in musculoskeletal conditions. Curr Med Res Opin, 2011; 27: 60522 .

Thatiparti, Thimma R, Shoffstall, Andrew J, Von Recum, Horst A. Cyclodextrin-based device coatings for affinity-based release of antibiotics. Biomaterials, 2010; 31: 2335-47.

Volobuef C, Moraes CM, Nunes LA, Cereda CM, Yokaichiya F, Franco MK, Braga AF, De Paula E, Tófoli GR, Fraceto LF, De Araujo DR. Sufentanil-2-hydroxypropyl- $\beta$-cyclodextrin inclusion complex for pain treatment: physicochemical, cytotoxicity, and pharmacological evaluation. J Pharm Sci, 2012; 101: 3698-707.

\section{How to cite this article:}

Manikandan A, Nemani SC, Sadheeshkumar V, Arumugam S. Spectroscopic Investigations for Photo Stability of Diclofenac Sodium Complexed with Hydroxypropyl-B-Cyclodextrin. J App Pharm Sci, 2016; 6 (04): 098-103. 